\title{
19. THE INTERPRETATION OF RECENT 21-CM LINE DATA IN TERMS OF LARGE-SCALE GALACTIC STRUCTURE
}

\author{
G. Westerhout \\ University of Maryland
}

The detection of the $21-\mathrm{cm}$ line 12 years ago opened up a vast new field of galactic research. But even though a large amount of data was assembled in these 12 years, our knowledge of the large-scale structure of our Galaxy still remains fairly limited. Let us summarize a few of the major facts which seem well established.

1. Our Galaxy is somewhere between an Sb and an Sc spiral. (We don't really know whether it isn't barred!)

2. We have a fairly good idea about the rotational motions of our Galaxy, at least in the inner parts. Between $R=3$ and $10 \mathrm{kpc}, 21-\mathrm{cm}$ line observations have yielded values which are estimated to be correct to within $\pm 5 \%$.

3. The hydrogen is distributed in a very thin and very flat plane, which, however, curves upwards in the northern hemisphere and downwards in the southern hemisphere.

4. The motions in the region of the galactic nucleus are very complicated and seem to indicate some expansion.

5. Individual cloud aggregates may have motions deviating from circular motion by as much as $20 \mathrm{~km} / \mathrm{sec}, 10 \mathrm{~km} / \mathrm{sec}$ probably being quite common.

6. Within 3 or $4 \mathrm{kpc}$ from the Sun the shape of the spiral arms agrees roughly with that of the spiral structure found in optically observable objects. But there seems to be considerable disagreement in position.

There remain, however, a large number of open questions. We list a few of them:

1. The difference between the northern and southern hemisphere rotation curves: large-scale deviations from circular motion.

2. Local deviations from circular motion.

3. Are the gas arms really spirals, or could they be rings?

4. What is the influence of the magnetic field?

5. What does the interstellar medium really look like: cloud structure, turbulence, kinetic temperature?

About the most important question to date is whether there is any large-scale deviation from circular motion such as expansion or streaming of gas inwards through the spiral arms. The observed difference between the rotation curves in the inner parts of the Galaxy in the northern and southern hemispheres finds its simplest explanation in the assumption that the "local standard of rest" is expanding. But since the observed solar motion with respect to the older stars is not appreciably different from that with respect to the gas and young stars, this would mean that a large proportion of the mass in the solar neighbourhood is moving outward. Such a disturbance in an average circular mass motion is of course not a priori impossible. 
Likewise, real deviations from circular motions in those parts of the Galaxy at which the $21-\mathrm{cm}$ rotational velocities were determined cannot be excluded either. Such deviations would point to rather considerable departures from a smooth axiallysymmetrical mass distribution.

Of course the whole question of the motion of the gas and what determines it is still virtually unanswered. The expanding motion of the gas closer to the nucleus might be tied in some way to the motions further out, and connected with the formation of the spiral arms. The suggestion has even been made that our Galaxy is a barred spiral and that the expanding motions near the centre are the radial components of streaming motions along the bar; it might also explain the differences between the northern and southern rotation curves. But so far, dynamics of barred spirals are little understood and the existence of a bar is only hypothetical.

The only things we can measure are radial velocities, and in some instances, rough distances. For example, assuming that the spiral arms have a constant thickness, one could determine their distance by measuring their angular extent in latitude. As another example, spiral arms seen in absorption must be in front of a bright source of continuum emission, and thus could be located approximately if the distance to the source were known. The latter method of distance determination, if worked out in detail and applied to observations with large telescopes which can see a considerable number of sources, might in the future prove to be of some value. But on the whole, these methods give large uncertainties.

Therefore, distance estimates can be made only if the laws of galactic rotation are known. As concluded above, this is not yet the case. In fact, even if they were known, large uncertainties would still remain, owing to peculiar motions of cloud aggregates or parts of spiral arms. Random deviations from circular velocity of the order of $10 \mathrm{~km} / \mathrm{sec}$ are probably quite common for fairly large hydrogen masses. Within $20^{\circ}$ from the centre and anticentre (i.e. along about $10 \%$ of the galactic equator) such random deviations, which are usually not detected as such, will result in erroneous distances from the Sun of the order of $1 \mathrm{kpc}$. At most other longitudes, distance errors due to this cause can easily amount to $500 \mathrm{pc}$. In particular near the Sun, within $2 \mathrm{kpc}$ or so, these errors are percentage-wise very large and make comparison with optical data very difficult, if not impossible.

In comparisons with optical data, the origin of the distance scale of the $21-\mathrm{cm}$ maps should be borne in mind. So far, all hydrogen maps have been produced with $A=19.5 \mathrm{~km} / \mathrm{sec} . \mathrm{kpc}$ and $R_{0}=8 \cdot 2 \mathrm{kpc}$. It has become increasingly clear that $A=15$ and $R_{0}=10$ are probably closer to the true values. The use of these latter values will mainly result in an increase in scale of most of the galactic hydrogen map by a factor 1·3. One should, in comparing optical with radio data, take this factor into account, and perhaps use the $A$ and $R_{0}$ one has just derived from his own optical data to scale the hydrogen data.

But even then, attempts to correlate hydrogen distances with the distribution of optical objects should be treated with caution, and the possibility of 0.5 to $1 \mathrm{kpc}$ errors in the radio distances should be considered all the time. Also, in making such comparisons, it would be advisable to use the actual contour maps of the distribution of neutral hydrogen, rather than the so-called "artist's impression". 
The contour maps represent what one can, with certain assumptions, derive by means of calculations from the line profiles. The "artist's impression" was made to display on a qualitative basis whatever details in the line profiles seemed significant, without putting much emphasis on actual densities, and using a certain amount of imagination in connecting various features.

One thing that should be borne in mind is that the spiral arms we see in other galaxies are composed of young stars and the associated ionized hydrogen. So far individual spiral arms in other galaxies have not yet been fully traced in the $21-\mathrm{cm}$ line. I think we should not a priori assume that the neutral hydrogen and the young stars are in exactly the same place. The gas motion must be governed to some extent by the magnetic fields and it might well be that the stars are removed from the gas arms fairly soon after their birth. The gas ionized by them is usually not typical of the general spiral arms medium, but rather denser.

There are two more points we should briefly mention in this survey of problems. The first one is the question whether the spiral arms should really be considered as continuous features. In many galaxies, the arms have a rather broken-up appearance when studied in detail, and one finds that only the first glance gives an overall impression of complete spirals. Our own Galaxy is no exception. Indeed, the three major arms can be followed over almost three-quarters of the circle in rough outline. But in detail, regions of high and low density alternate over distances of the order of $1 \mathrm{kpc}$ along the arms, and the connections in many places are doubtful. I would almost tend to describe the structure as a somewhat loose assembly of elongated concentrations of hydrogen, usually arranged along circular arcs, but sometimes rather inclined with respect to circles around the centre.

The final problem is that of the hydrogen densities. This is directly connected with the question of the gas temperature and the structure of the individual clouds. Do the clouds consist mainly of small high-density regions, of large clouds with low density, of a combination of these, or do we have to see clouds as filaments or sheets similar to the dust sheets in the Pleiades? In the $21-\mathrm{cm}$ line reductions, individual clouds were assumed to have low optical depths, and the gas temperature was assumed to be constant throughout the Galaxy. The corrections for random cloud velocities were very uncertain. The large uncertainties in these assumptions introduce uncertainties in the density in particular regions and thus in the overall density and hydrogen mass. These uncertainties could well be of the order of 50 to $100 \%$, probably in the sense of underestimation. The average density of neutral hydrogen in the galactic plane out to a few kiloparsecs beyond the Sun is somewhere between 0.5 and $1.0 \mathrm{~cm}^{-3}$, with the interarm densities lower than the densities in the arms by at least a factor of 2 , probably much more. The total mass of hydrogen might be anywhere between 0.2 and $0.5 \times 10^{10} M_{\odot}$.

So far, our discussion of the large-scale structure of the Galaxy has rested mainly on the two surveys at Leiden and Sydney, made with relatively small instruments many years ago. It might well be that new surveys, providing a more complete coverage of the Milky Way, will enable us to follow certain details in the structure much more closely and thereby get some ideas of deviations from circular motion. Such surveys are under way in a few places at present. Very high-reso- 
lution studies are probably necessary to solve the problem of the structure of the individual clouds and the temperature of the neutral hydrogen. Such high-resolution studies will also give the opportunity of studying more features in absorption and thus perhaps give some rough distance estimates. Clearly, we have made only the first step in the $21-\mathrm{cm}$ line research of the structure of our Galaxy.

\section{HIGH-RESOLUTION STUDIES OF THE GALACTIC EQUATOR REGION}

\section{F. J. KERR}

\section{CSIRO Radiophysics Laboratory, Sydney}

A large program of observations is being undertaken with the 210 -foot telescope at Parkes, to study the fine structure of the $21-\mathrm{cm}$ line emission in the region of the galactic equator. With the aid of a multi-channel receiver, $\mathrm{H}$-line profiles have been obtained every $6 \mathrm{~min}$ of arc (i.e. every half-beamwidth) along the equator from $l \mathrm{II}=300$ to $60^{\circ}$, and also from $b^{\mathrm{II}}=-3$ to $+3^{\circ}$ along a number of constantlongitude lines. The bandwidth of each channel of the receiver is equivalent to $8 \cdot 0$ $\mathrm{km} / \mathrm{sec}$, with a spacing of $7.0 \mathrm{~km} / \mathrm{sec}$ between the centres of adjacent channels. The data have been recorded on punched paper tape, and the main reduction is being carried out on the SILLIAC computer of the University of Sydney. A sample group of profiles, illustrating the great variability from point to point, has been published previously (Kerr 1962b).

In addition to the great increase in resolving power, the present survey has the advantage over the previous combined observations in that the regions north and south of the galactic centre are being studied with the same instrument and the same techniques. The very detailed results have been only partially reduced so far, but a number of conclusions can be presented.

\section{The Hydrogen Layer}

The thickness of the hydrogen layer between half-density levels can be derived from observations in the tangential-point regions. With the higher resolution, the thickness is found to be about $170 \mathrm{pc}$ in the region between 3 and $7 \mathrm{kpc}$ from the galactic centre, rather than the earlier $21-\mathrm{cm}$ value of $220 \mathrm{pc}$. The lower figure agrees better with values of layer thickness derived for cepheids and HII regions.

The new observations contirm the extreme flatness of the hydrogen layer, as indicated by the earlier lower-resolution surveys. Over the whole region inside a circle through the Sun, the deviations of the main layer from a plane are always less than a few tens of parsecs. A more precise solution for the galactic plane will result from the present survey.

\section{Detail in a Spiral Arm}

An interesting question connected with galactic spiral structure is the degree of continuity along an individual spiral arm. A detailed examination has been made of the "far outer arm", which is a well-marked feature on the far side of the Galaxy north of the centre. This arm was chosen for study because it can be seen from 